\title{
Optimal Decentralized LQR Control to Enhance Multi-Area LFC System Stability
}

\author{
Ashraf M. Abdelhamid ${ }^{1}{ }^{1}$ and Ahmed A. M. El-Gaafary ${ }^{2}$ \\ ${ }^{1}$ Electronics and Communications Engineering Department, College of Engineering, Umm Al-Qura University, \\ Al-Lith Branch, Saudi Arabia \\ ${ }^{2}$ Electrical Engineering Department, Faculty of Engineering, Minia University, Minya, Egypt \\ Correspondence should be addressed to Ashraf M. Abdelhamid; ashrafmed@hotmail.com
}

Received 12 December 2017; Revised 8 March 2018; Accepted 1 April 2018; Published 12 August 2018

Academic Editor: Yurong Liu

Copyright (C) 2018 Ashraf M. Abdelhamid and Ahmed A. M. El-Gaafary. This is an open access article distributed under the Creative Commons Attribution License, which permits unrestricted use, distribution, and reproduction in any medium, provided the original work is properly cited.

\begin{abstract}
Many studies have been made in the field of load frequency control (LFC) through the last few decades because of its importance to healthy power system. It is important to maintain frequency deviation at zero level after a load perturbation. In decentralized control, the multi-area power system is decomposed into many single input single output (SISO) subsystems and a local controller is designed for each subsystem. The controlled subsystems may have slow poles; these undesired poles may drive the aggregated overall system into the instability region. Thus, it is required to relocate these poles to much more stable places to avoid their effect upon the overall system stability. This study aims to design a new load frequency controller based on the powerful optimal linear quadratic regulator (LQR) technique. This technique can be applied over subsystem level to shift each subsystem undesired poles one by one into a prespecified stable location which in turn shift the overall system slow poles and reduce the effect of the interaction between the interconnected subsystems among each other. This procedure must be applied many times as the number of undesired poles (pairs) until all the desired poles are achieved. The main objective is considered to get a robust design when the system is affected by a physical disturbance and $\pm 40 \%$ model uncertainties. LQR can be applied again over the aggregated system to enhance the stability degree. Simulation results are used to evaluate the effectiveness of the proposed method and compared to other research results.
\end{abstract}

\section{Introduction}

LFC is one of the most important problems in electric power system design/operation and is becoming much more significant nowadays especially with the increasing dimensions and complexity of the interconnected power systems. The decentralized control strategies are widely used recently because of the ability of using the traditional local control methodologies [1]. In the decentralized control, the large-scale power system is divided into many separated subsystem models and so the complex control problem can be decomposed into individual subproblems that have some interactions among each other. Currently the classical control techniques can be easily applied over subsystem level [2]. Each subsystem contains a local controller which can be of different type and has access to local sensors only [2]. These all independent controllers together represent a decentralized controller, and the failure of one local controller does not necessarily lead to overall system instability.

Large-scale power systems usually consist of many control areas, connected by tie-lines to exchange the power demands among them [5]. Loads at any station can appear randomly and changeable so that the system frequency and tieline power will deviate from their nominal values [6]. There has been continuing interest in designing load frequency controllers with better performance to maintain the frequency and to keep tie-line power flows within desired values by using various decentralized control methods during the last two decades. In [7] the author has designed local independent $H_{\infty}$ controllers to decentralized two-area power system. He has used a condition based on the structured singular values (SSVs). Also, in [8] local independent LFCs have been designed for interconnected two-area power systems to realize satisfactory performances based on the SSV $\mu$ 
methods. Sequential decentralized LFCs for three area power systems based on $\mu$ synthesis has been designed, and system uncertainties and practical constraints have been considered [9]. In [10], the same methodology has been applied to stabilize four area power systems. The paper [3] has mixed $\mathrm{H}_{2}$ and $\mathrm{H}_{\infty}$ control methods and has considered the connections between each area and the rest of the system as new disturbance. In [4], Particle Swarm Optimization (PSO) has been used in the AC-DC interconnected LFC system. Local controller to each area has been designed to stabilize the overall system and reduce settling time and frequency oscillation.

A change in real power demand at any point of a network is reflected throughout the system by a change in frequency. Therefore, system frequency provides a good indicator to system generation and load imbalance $[11,12]$.

Linear quadratic optimal systems based on predetermined eigenvalues are an important manner. Alternative methods are developed to construct the weighting matrix $Q$ that enables the user to shift undesired system eigenvalues (unstable or slow) into prespecified (stable or faster) ones. The process can be repeated many times until all the desired pole locations are placed as desired. The paper [13] has developed a method to find the weighting matrix element dependent on the desired performance and the prescribed eigenvalues. The authors in [14] have introduced a methodology to achieve the prescribed eigenvalue locations using LQR to improve the undesired eigenvalues (slow or undampened) to faster and dampened ones. Closed loop poles and system performance have been combined in [15] as an objective function to find the optimal weighting matrices for LQR using intelligent optimization techniques. Also, [16] has presented an algorithm to determine the weighting matrices of the LQR according to the desired poles placement.

1.1. Research Objectives. The main idea of this work is to propose an optimal controller based on LQR approach and enable the system to beat specified uncertainties caused by variations of inertia constant $\left(M_{i}\right)$ and tie-line synchronizing coefficient between areas $i$ and $j\left(T_{i j}\right)$, parameters of all control areas, and load disturbances as follows:

(i) Maintaining system stability and performance for the overall power system and each control area throughout $\pm 40 \%$ uncertainty of $M_{i}$ and $T_{i j}$

(ii) Minimizing the effectiveness of step load disturbance on output signals to zero

(iii) Recognizing a minimum overshoot and undershoot values

(iv) Minimizing settling time on the frequency deviation signal and obtaining zero-steady state error for individual control area

\subsection{Design Methodology}

(i) The first step is to divide the complex Multi-Input Multi-Output (MIMO) problem into simple SISO subproblems.

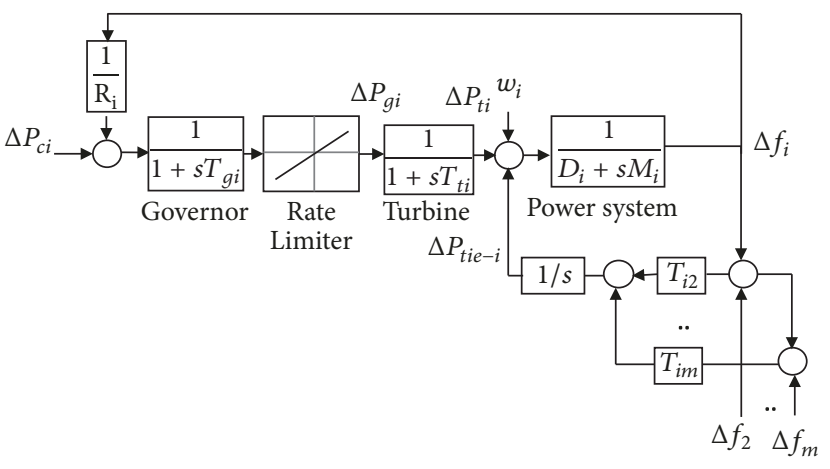

Figure 1: Block diagram of control area-1 [3, 4].

(ii) LQR technique is used to design a local controller for each decoupled subsystem to realize design objectives.

(iii) Slower eigenvalues are shifted more inside the stability region within prespecified values.

(iv) The controlled system is composed again, and the overall stability is determined.

(v) LQR is applied again over the aggregated overall system level to enhance system stability.

(vi) The proposed control methodology is testified and compared to other research results.

\section{Model Description}

In this study, the conventional model is used for each control area of a multi-area power system which is widely used in many studies $[3,4,10]$. The same system parameters are used in [10] for the three areas and in [3] for the four area. Referring to the simplified traditional-based LFC model shown in Figure 1 for control area 1 , the state space realization of area $i$ (from 4-control area power system) is given by

$$
\begin{aligned}
& \dot{x}_{i}=A x_{i}+B u_{i}+w_{i} d_{i} \\
& y_{i}=C_{i} x_{i}
\end{aligned}
$$

The state vector $x_{i}$, control input $u_{i}$, disturbance input $w_{i}$, and the measured output $y_{i}$ can be defined as

$$
x_{i}=\left[\begin{array}{c}
\Delta f_{i} \\
\Delta P_{t i} \\
\Delta P_{g i} \\
\Delta P_{t i e-i}
\end{array}\right] \text {, }
$$

$$
\begin{aligned}
& u_{i}=\Delta P_{c i}, \\
& w_{i}=\Delta P_{d i}, \\
& y_{i}=\beta_{i} \Delta f_{i}+\Delta P_{t i e-i}
\end{aligned}
$$


Then, the nominal state space model for a 4-area power system can be described by (3) as

$x(t)$

$$
=\left[\begin{array}{lllllllll}
\Delta f_{1} & \Delta P_{t 1} & \Delta P_{g 1} & \Delta P_{t i e-1} & \cdots & \Delta f_{4} & \Delta P_{t 4} & \Delta P_{g 4} & \Delta P_{t i e-4}
\end{array}\right]^{T}
$$$$
u(t)=\left[\begin{array}{llll}
u_{1} & u_{2} & u_{3} & u_{4}
\end{array}\right]^{T}
$$

$$
d(t)=\left[\begin{array}{llll}
\Delta P_{d 1} & \Delta P_{d 2} & \Delta P_{d 3} & \Delta P_{d 4}
\end{array}\right]^{T}
$$$$
y(t)=\left[\begin{array}{llll}
y_{1} & y_{2} & y_{3} & y_{4}
\end{array}\right]^{T}
$$

where

$\Delta f_{i}$ is frequency deviation of area $i$,

$\Delta P_{g i}$ is governor valve position of generator in area $i$,

$\Delta P_{c i}$ is governor load set point in area $i$,

$\Delta P_{t i}$ is turbine power in area $i$,

$\Delta P_{\text {tie-i } i}$ is change in net tie-line power flow in area $i$,

$\Delta P_{d i}$ is disturbance in area $i$,

$M_{i}$ is equivalent inertia constant for area $i$,

$D_{i}$ is equivalent damping coefficient for area $i$,

$T_{g i}$ is governor time constant for area $i$,

$T_{t i}$ is turbine time constant for area $i$,

$T_{i j}$ is tie-line synchronizing coefficient between areas $i$ and $j$,

$R_{i}$ is drooping characteristic for area $i$,

in which

$$
\begin{aligned}
& A \in R^{16 \times 16}, \\
& B \in R^{16 \times 4}, \\
& W \in R^{16 \times 4}, \\
& C \in R^{4 \times 16} \\
& A=\left[\begin{array}{llll}
A_{11} & A_{12} & A_{13} & A_{14} \\
A_{21} & A_{22} & A_{23} & A_{24} \\
A_{31} & A_{32} & A_{33} & A_{34} \\
A_{41} & A_{42} & A_{43} & A_{44}
\end{array}\right]
\end{aligned}
$$

$$
\begin{aligned}
A_{i i} & =\left[\begin{array}{cccc}
-\frac{D_{i}}{M_{i}} & \frac{1}{M_{i}} & 0 & -\frac{1}{M_{i}} \\
0 & -\frac{1}{T_{t i}} & \frac{1}{T_{t i}} & 0 \\
-\frac{1}{R_{i} T_{g i}} & 0 & -\frac{1}{T_{g i}} & 0 \\
\sum_{j} T_{i j} & 0 & 0 & 0
\end{array}\right], \\
A_{i j}(i \neq j) & =\left[\begin{array}{cccc}
0 & 0 & 0 & 0 \\
0 & 0 & 0 & 0 \\
0 & 0 & 0 & 0 \\
-T_{i j} & 0 & 0 & 0
\end{array}\right]
\end{aligned}
$$

and

$$
\begin{aligned}
& B_{1}=\left[\begin{array}{cccc}
0 & 0 & 0 & 0 \\
0 & 0 & 0 & 0 \\
-\frac{1}{T_{g 1}} & 0 & 0 & 0 \\
0 & 0 & 0 & 0
\end{array}\right], \\
& B_{2}=\left[\begin{array}{cccc}
0 & 0 & 0 & 0 \\
0 & 0 & 0 & 0 \\
0 & -\frac{1}{T_{g 2}} & 0 & 0 \\
0 & 0 & 0 & 0
\end{array}\right], \\
& B_{3}=\left[\begin{array}{llll}
0 & 0 & 0 & 0 \\
0 & 0 & 0 & 0 \\
0 & 0 & -\frac{1}{T_{g 3}} & 0 \\
0 & 0 & 0 & 0
\end{array}\right], \\
& B_{4}=\left[\begin{array}{llll}
0 & 0 & 0 & 0 \\
0 & 0 & 0 & 0 \\
0 & 0 & 0 & -\frac{1}{T_{g 4}} \\
0 & 0 & 0 & 0
\end{array}\right]
\end{aligned}
$$

and

$$
\begin{aligned}
& W_{1}=\left[\begin{array}{cccc}
-\frac{1}{M_{1}} & 0 & 0 & 0 \\
0 & 0 & 0 & 0 \\
0 & 0 & 0 & 0 \\
0 & 0 & 0 & 0
\end{array}\right], \\
& W_{2}=\left[\begin{array}{cccc}
0 & -\frac{1}{M_{2}} & 0 & 0 \\
0 & 0 & 0 & 0 \\
0 & 0 & 0 & 0 \\
0 & 0 & 0 & 0
\end{array}\right]
\end{aligned}
$$


TABLE 1: Nominal power system parameter values.

\begin{tabular}{|c|c|c|c|c|c|}
\hline & $\begin{array}{c}D_{i} \\
\text { p.u.MW/Hz } \\
\end{array}$ & $\begin{array}{c}M_{i} \\
\text { p.u.MW }\end{array}$ & $\begin{array}{l}T_{t i} \\
\text { Sec } \\
\end{array}$ & $\begin{array}{l}T_{g i} \\
\text { Sec } \\
\end{array}$ & $\begin{array}{c}R_{i} \\
\text { Hz/p.u.MW }\end{array}$ \\
\hline Area 1 & 0.0083 & 0.166 & 0.3 & 0.08 & 2.4 \\
\hline Area 2 & 0.0088 & 0.222 & 0.33 & 0.072 & 2.7 \\
\hline Area 3 & 0.0080 & 0.16 & 0.35 & 0.07 & 2.5 \\
\hline Area 4 & 0.0088 & 0.13 & 0.375 & 0.085 & 2 \\
\hline $\begin{array}{l}T_{i j} \\
\text { p.u.MW/Hz }\end{array}$ & \multicolumn{4}{|c|}{$T_{12}=T_{13}=T_{14}=T_{21}=T_{23}=T_{31}=T_{32}=T_{41}=0.545$} & \\
\hline
\end{tabular}

TABLE 2: Parameter variation limits lead to system instability.

\begin{tabular}{cc}
\hline $\boldsymbol{T}_{\boldsymbol{i} j}$ & $T_{12} \geq 0.645, T_{13}, T_{14} \geq 0.605, T_{32} \geq 0.615, T_{21}, T_{23} \leq 0.475$, and $T_{31} \leq 0.445$. \\
\hline $\boldsymbol{M}_{\boldsymbol{i}}$ & $M_{1} \leq 0.056, M_{2}, M_{3}$, and $M_{4} \leq$ its nominal values \\
\hline
\end{tabular}

$$
\begin{aligned}
& W_{3}=\left[\begin{array}{cccc}
0 & 0 & -\frac{1}{M_{3}} & 0 \\
0 & 0 & 0 & 0 \\
0 & 0 & 0 & 0 \\
0 & 0 & 0 & 0
\end{array}\right], \\
& W_{4}=\left[\begin{array}{lllc}
0 & 0 & 0 & -\frac{1}{M_{4}} \\
0 & 0 & 0 & 0 \\
0 & 0 & 0 & 0 \\
0 & 0 & 0 & 0
\end{array}\right]
\end{aligned}
$$

(6)

The total real power imported to area $i$ equals the sum of all inflowing line powers $\Delta P_{t i e-i j}$ from adjoining areas, i.e.,

$$
P_{t i e-i}=\sum_{j} P_{t i e-i j}
$$

The real power in per unit transmitted across a lossless line of reactance $X_{i j}$ is

$$
\begin{aligned}
P_{t i e-i j} & =\frac{\left|V_{i}\right|\left|V_{j}\right|}{X_{i j} P_{r i}} \sin \left(\theta_{i}-\theta_{j}\right) \\
V_{i} & =\left|V_{i}\right| e^{j \theta_{i}}
\end{aligned}
$$

where

$$
P_{r i} \text { is the rated power of area } i \text {, }
$$

$V_{i}$ and $\theta_{i}$ are the amplitude and angle of the terminal voltage in area $i$.

A simplified 4-area power system is represented in Figure 2 with system nominal values as illustrated in Table $1[3,11]$. These values make the 4-area open loop power system stable, but the open loop system performance is affected by changes in the equivalent inertia constants $M_{i}$ and synchronizing coefficients $T_{i j}$ (Table 2) more than changes of other parameters; these parameter values may lead the system into the instability region.

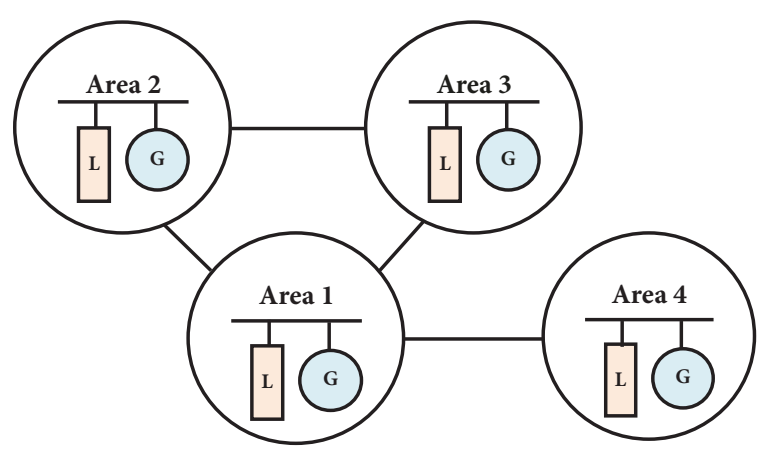

FIgURE 2: Simplified 4-area power system.

\section{LQR Technique}

LQR is an optimal design technique which guarantees robustness, while pole placement technique gives the desired performance but does not guarantee robustness. In this procedure, a design of linear quadratic system with prescribed poles has been followed and applied to shift slow decoupled subsystem eigenvalues to defense the effect of the interconnections between subsystems that leads to much more stable overall system poles and enhances the overall system stability. This procedure was developed by Solheim [13].

The optimal gain is determined to minimize the cost function $J$ as follows:

$$
J=\frac{1}{2} \int_{0}^{\infty}\left(x^{T} Q x+u^{T} R u\right) d t
$$

where $R$ and $Q$ are the weighting matrices of the input $u(t)$ and states $x(t)$, respectively [17], and the control gain $k$ is given by

$$
k=R^{-1} B^{T} P
$$

where $P$ is the unique, symmetric, positive definite solution to steady state Algebraic Riccati Equation (ARE)

$$
P A+A^{T} P-P B R^{-1} B^{T} P+Q=0
$$


To construct the weighting matrix $Q$ to shift the complex conjugate poles to complex places, (13) is used with the constraint in (14) for its internal elements $\widetilde{q}$

$$
\begin{aligned}
\widetilde{q}_{11}= & \frac{2\left(\sigma^{2}-\alpha^{2}\right)-2\left(\omega^{2}-\beta^{2}\right)}{h_{11}+h_{22}} \\
\left(\sigma^{2}+\omega^{2}\right)^{2}= & \left(\alpha^{2}+\beta^{2}\right)^{2}+\widetilde{q}_{11}\left(h_{11}+h_{22}\right)\left(\alpha^{2}+\beta^{2}\right) \\
& +\widetilde{q}_{11}{ }^{2}\left(h_{11} h_{22}-{h_{12}}^{2}\right)
\end{aligned}
$$

where

$h$ are the elements of the solution of the matrix $(H=$ $M^{-1} B P^{-1} B^{T} M^{-T}$ ) and $M$ is an eigenvector matrix corresponding to the system matrix $A$,

$\alpha \pm j \beta$ are the old complex eigenvalues and $\sigma \pm j \omega$ are the desired complex eigenvalues.

If it is required to shift complex conjugate poles to real places, (15) is used with the constraint in (16)

$$
\begin{aligned}
\widetilde{q}_{11}= & \frac{\sigma_{1}^{2}+\sigma_{2}^{2}-2\left(\alpha^{2}-\beta^{2}\right)}{h_{11}+h_{22}} \\
\sigma_{1}{ }^{2} \sigma_{2}{ }^{2}= & \left(\alpha^{2}+\beta^{2}\right)^{2}+\widetilde{q}_{11}\left(h_{11}+h_{22}\right)\left(\alpha^{2}+\beta^{2}\right) \\
& +\widetilde{q}_{11}{ }^{2}\left(h_{11} h_{22}-h_{12}{ }^{2}\right)
\end{aligned}
$$

while if it is required to shift the real eigenvalues to real places, (17) is used

$$
\tilde{q}_{j j}=\frac{S_{j}^{2}-\lambda_{j}^{2}}{h_{j j}}
$$

where $S_{j}$ is the desired real eigenvalue and $\lambda_{j}$ is the old real one.

\section{Controller Design}

The decentralized input control methodology presented in [18] is used in order to decompose the system into four decoupled subsystems $\left(A_{i}\right)$ with interconnected subsystems $\left(A_{i j}\right)$.

$$
\dot{x}_{i}=A_{i} x_{i}+\sum_{\substack{j=1 \\ j \neq i}}^{s} A_{i j} x_{j}+b_{i} u_{i}, \quad i=1,2, \ldots, s
$$

This system can be stabilized by a multilevel control. Each decoupled subsystem can be stabilized separately by using local controller, which can provide a desired degree of stability, while global controllers are applied to enhance the overall system stability through reducing the effect of the interconnections among subsystems [18-20], with control effort as

$$
u_{i}=u_{i}^{l}(t)+u_{i}^{g}(t)
$$

where $u_{i}^{l}(t)$ is the local controller and chosen as

$$
u_{i}^{l}(t)=-k_{i}^{T}(t) x_{i}
$$

With a constant vector $k_{i} \in R^{n_{i}}, u_{i}{ }^{g}(t)$ is the global controller and chosen as

$$
u_{i}^{g}(t)=-\sum_{j=1}^{s} k_{i j}^{T} x_{j}
$$

where $k_{i j} \in R^{n_{j}}$ are constant vectors.

To start stabilization process, (18) must be represented in canonical form

$$
\dot{x}_{i}{ }^{c}=A_{i}{ }^{c} x_{i}{ }^{c}+\sum_{\substack{j=1 \\ j \neq i}}^{s} A_{i j}{ }^{c} x_{j}{ }^{c}+b_{i}{ }^{c} u_{i}, \quad i=1,2, \ldots, s
$$

with appropriate transformations $Q_{i}{ }^{c}$ and $Q_{j}{ }^{c}$, where

$$
\begin{aligned}
A_{i}{ }^{c} & =\left(Q_{i}{ }^{c}\right)^{-1} A_{i} Q_{i}{ }^{c} \\
A_{i j}{ }^{c} & =\left(Q_{i}{ }^{c}\right)^{-1} A_{i j} Q_{j}{ }^{c} \\
B_{i}{ }^{c} & =\left(Q_{i}{ }^{c}\right)^{-1} B_{i} \\
C_{i}{ }^{c} & =C_{i} Q_{i}{ }^{c} \\
F_{i}{ }^{c} & =\left(Q_{i}{ }^{c}\right)^{-1} F_{i}
\end{aligned}
$$

Substituting (20) and (21) into (22) with the appropriate transformations (23) yields

$$
\begin{aligned}
\dot{x}_{i}{ }^{c}= & \left(A_{i}{ }^{c}-b_{i}^{c}\left(k_{i}^{c}\right)^{T}\right) x_{i}^{c} \\
& +\sum_{\substack{j=1 \\
j \neq i}}^{s}\left(A_{i j}{ }^{c}-b_{i}^{c}\left(k_{i j}{ }^{c}\right)^{T}\right) x_{j}{ }^{c}, \quad i=1,2, \ldots, s
\end{aligned}
$$

A linear nonsingular transformation matrix $T_{i}$ is applied to (24) to diagonalize it as follows:

$$
\begin{array}{rl}
A_{i j}{ }^{d} & =T_{i}{ }^{-1} A_{i j}{ }^{c} T_{j} \\
b_{i}{ }^{d} & =T_{i}{ }^{-1} b_{i}{ }^{c} \\
\left(k_{i j}{ }^{d}\right)^{T} & =\left(k_{i j}{ }^{c}\right)^{T} T_{j} \\
\dot{\tilde{x}}_{i}{ }^{d} & =\Lambda_{i}{\widetilde{x_{i}}}^{d}+\sum_{\substack{j=1 \\
j \neq i}}^{s}\left(A_{i j}{ }^{d}-b_{i}{ }^{d}\left(k_{i j}{ }^{d}\right)^{T}\right){\widetilde{x_{j}}}^{d}, \\
i & i=1,2, \ldots, s
\end{array}
$$

where $k_{i j}{ }^{d}$ can be calculated from (27) to reduce the effect of the interconnected subsystems.

$$
k_{i j}{ }^{d}=\left[\left[\left(b_{i}{ }^{d}\right)^{T}\left(b_{i}^{d}\right)\right]^{-1}\left[\left(b_{i}^{d}\right)^{T}\left(A_{i j}{ }^{d}\right)\right]\right]^{T}
$$


The Overall Controller Gain. Through the process of stabilizing the MIMO system, the feedback control vector can be calculated using $k_{i}$ and $k_{i j}$ which computed for subsystem level. To obtain the control function $u(t)$, substitute (20) and (21) into (19), which yields

$$
\begin{aligned}
& u_{i}(t)=-\left(k_{i}^{c}\right)^{T} x_{i}^{c}-\sum_{\substack{j=1 \\
j \neq i}}^{s}\left(k_{i j}{ }^{d}\right)^{T} T_{j}{ }^{-1} x_{j}{ }^{c} \\
& u_{i}(t)=-\left[\left(k_{i}^{c}\right)^{T}+\sum_{\substack{j=1 \\
j \neq i}}^{s}\left(k_{i j}{ }^{d}\right)^{T} T_{j}{ }^{-1}\right] x^{c}
\end{aligned}
$$

where

$$
k^{c}=\left[\left(k_{i}^{c}\right)^{T}+\sum_{\substack{j=1 \\ j \neq i}}^{s}\left(k_{i j}{ }^{d}\right)^{T} T_{j}^{-1}\right]
$$

and $k^{c}$ can be expressed in detail as

$$
k^{c}=\left[\begin{array}{cccc}
\left(k_{1}{ }^{c}\right)^{T} & \left(k_{12}{ }^{d}\right)^{T} T_{2}{ }^{-1} & \ldots & \left(k_{1 s}{ }^{d}\right)^{T} T_{s}{ }^{-1} \\
\left(k_{21}{ }^{d}\right)^{T} T_{1}{ }^{-1} & \left(k_{2}{ }^{c}\right)^{T} & \cdots & \left(k_{2 s}{ }^{d}\right)^{T} T_{s}{ }^{-1} \\
\vdots & \vdots & & \vdots \\
\left(k_{s 1}{ }^{d}\right)^{T} T_{1}^{-1} & \left(k_{s 2}{ }^{d}\right)^{T} T_{2}^{-1} & & \left(k_{s}^{c}\right)^{T}
\end{array}\right]
$$

4.1. Scenario I: Decentralized Input Controller Design with $L Q R$. Weighting function values for each area can be selected using trial and error after many trials to their best values of

$$
\begin{aligned}
& Q_{1}=\left[\begin{array}{cccc}
10 & 0 & 0 & 0 \\
0 & 1 & 0 & 0 \\
0 & 0 & 10 & 0 \\
0 & 0 & 0 & 1
\end{array}\right], \\
& R_{1}=0.1 \text {, } \\
& Q_{2}=\left[\begin{array}{cccc}
10 & 0 & 0 & 0 \\
0 & 1 & 0 & 0 \\
0 & 0 & 10 & 0 \\
0 & 0 & 0 & 10
\end{array}\right], \\
& R_{2}=0.01 \\
& Q_{3}=\left[\begin{array}{cccc}
10 & 0 & 0 & 0 \\
0 & 0.1 & 0 & 0 \\
0 & 0 & 1 & 0 \\
0 & 0 & 0 & 10
\end{array}\right],
\end{aligned}
$$

$$
\begin{aligned}
R_{3} & =0.1, \\
Q_{4} & =\left[\begin{array}{cccc}
10 & 0 & 0 & 0 \\
0 & 0.1 & 0 & 0 \\
0 & 0 & 1 & 0 \\
0 & 0 & 0 & 10
\end{array}\right], \\
R_{4} & =0.1
\end{aligned}
$$

Step responses to $0.01 \mathrm{p} . \mathrm{u}$ input and also to $0.01 \mathrm{p} . \mathrm{u}$ disturbance are illustrated in Figure 3. To examine the robustness of the design, Figure 4 shows step response of the controlled system with LQR to $-40 \%$ uncertainties, (a) to 0.01 p.u input and (b) to 0.01 p.u disturbance.

4.2. Scenario II: LQR Design with Prespecified Pole Locations. It is important to shift each area slow eigenvalue more inside the stability region to minimize the effect of their values and the effect of the interconnected subsystems on the overall system stability. Weighting functions $Q$ are calculated to realize this objective as discussed in Section 3.

This subsection shows designing a controller for subsystem-1 and so the other subsystems will follow the same procedures.

For Subsystem-1. Transform subsystem into controllable canonical form. For subsystem eigenvalues of $[-13.256-0.3534 \pm j 3.999-1.92]$, it is needed to start moving the slowest complex conjugate pair $(-0.3534 \pm j 3.999)$ more into the stability region to a new location of $(-6 \pm j 2.4)$.

Then, diagonalize the system with an appropriate transformation to the form:

$$
A_{1 c d}=10^{2} *\left[\begin{array}{cccc}
-13.256 & 0 & 0 & 0 \\
0 & -1.92 & 0 & 0 \\
0 & 0 & -0.35 & 4 \\
0 & 0 & -4 & -0.35
\end{array}\right]
$$

First the weighting matrix $\widetilde{Q}_{i}$ can be calculated as the form:

$$
\widetilde{Q}_{i}=\left[\begin{array}{cccc}
\widetilde{q}_{11} & 0 & 0 & 0 \\
0 & \widetilde{q}_{22} & 0 & 0 \\
0 & 0 & \widetilde{q}_{33} & 0 \\
0 & 0 & 0 & \widetilde{q}_{44}
\end{array}\right], \quad \widetilde{q}_{33}=\widetilde{q}_{44}
$$

From (13) and (14), $\widetilde{q}_{33}$ is calculated to be 118.6256. Move the second slow area-1 pole $(-1.92)$ to the new location of $(-20)$ using (17) with the value of $\widetilde{q}_{22}=50763.88$. The new area-1 eigenvalues become $[-13.255-20-6 \pm j 2.402 i]$, with final $\widetilde{Q}_{1}$ value of

$$
\widetilde{Q}_{1}=\left[\begin{array}{cccc}
1 & 0 & 0 & 0 \\
0 & 50763.88 & 0 & 0 \\
0 & 0 & 118.6256 & 0 \\
0 & 0 & 0 & 118.6256
\end{array}\right]
$$




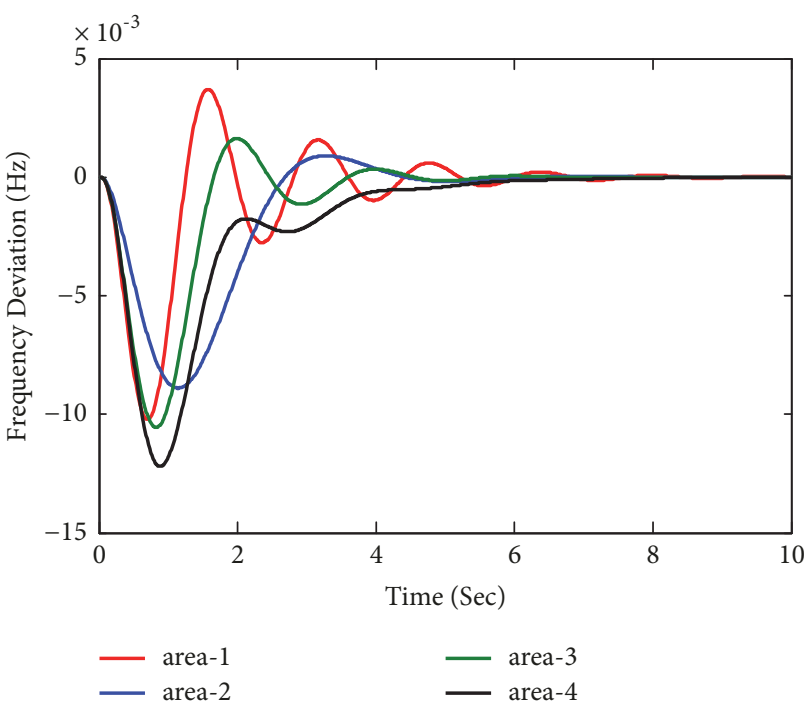

(a)

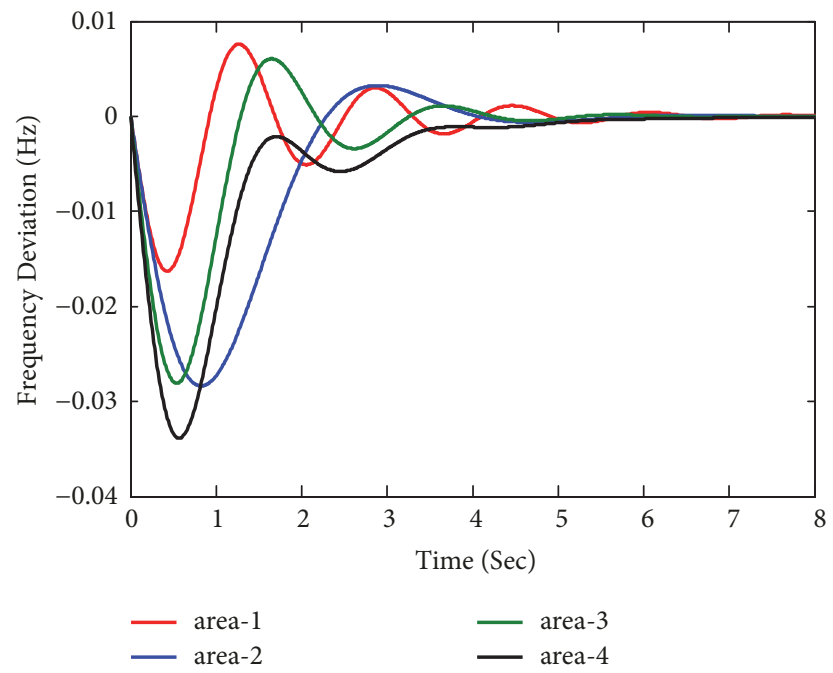

(b)

FIGURE 3: Step response of the controlled system for scenario I, to (a) 0.01 p.u. input and (b) 0.01 disturbance input.

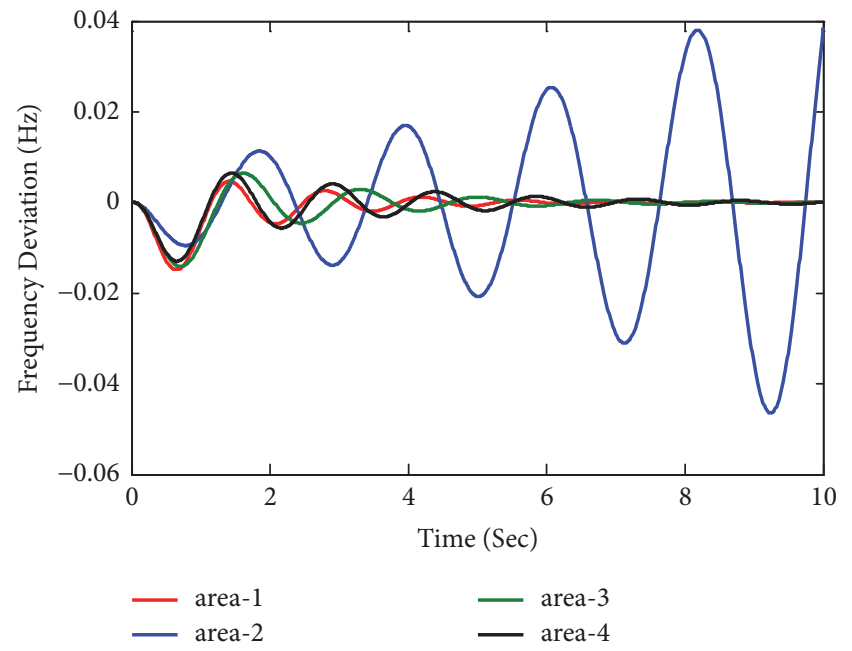

(a)

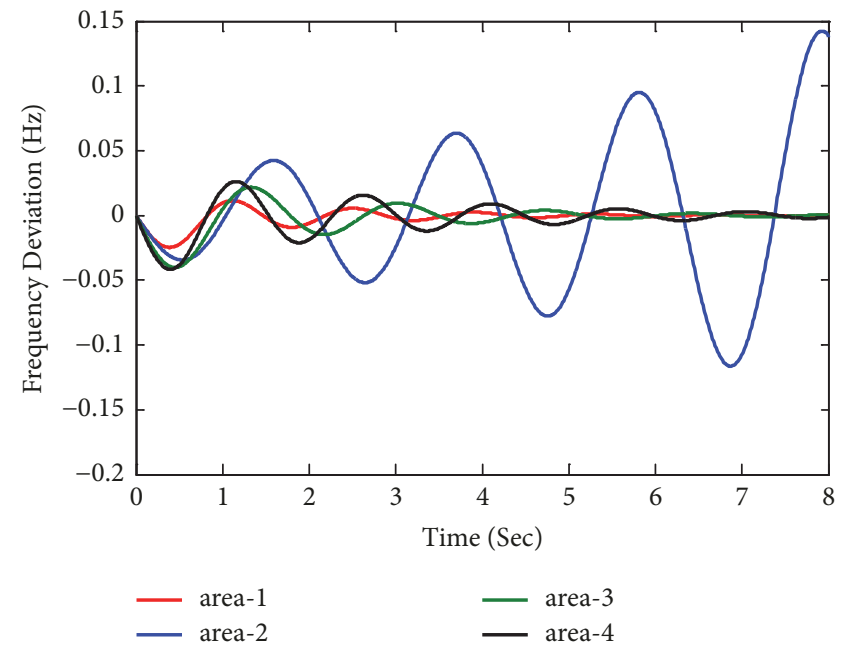

(b)

FIGURE 4: Step response of the 4 areas in scenario I with $-40 \%$ uncertainties of $M_{i}$, and $T_{i j}$ to (a) 0.01 p.u input and (b) 0.01 p.u disturbance.

Table 3 summarizes original and achieved pole locations for each area with the calculated weighting matrix $Q$.

Local controllers are determined to be equal to

$$
\begin{aligned}
k_{a} & =\left[\begin{array}{llll}
10660 & 4307 & 653 & 29.4
\end{array}\right] \\
k_{b} & =\left[\begin{array}{llll}
5497 & 2820 & 533 & 25
\end{array}\right] \\
k_{c} & =\left[\begin{array}{llll}
7300 & 3100 & 560 & 26
\end{array}\right] \\
k_{d} & =\left[\begin{array}{llll}
6726 & 2850 & 536 & 27.8
\end{array}\right]
\end{aligned}
$$

Global controllers are calculated to reduce the effect of the interaction between the interconnected subsystems to be

$$
\begin{aligned}
& K_{12}=\left[\begin{array}{llll}
-0.268 & -0.523 & -3.26 & -4.28
\end{array}\right] \\
& K_{13}=\left[\begin{array}{llll}
-0.268 & -0.523 & -3.26 & -4.28
\end{array}\right]
\end{aligned}
$$

$$
\begin{aligned}
& K_{14}=\left[\begin{array}{llll}
-0.36 & -0.654 & -1.794 & -5.36
\end{array}\right] \\
& K_{21}=\left[\begin{array}{llll}
-0.353 & -0.804 & -2.447 & -2.33
\end{array}\right] \\
& K_{23}=\left[\begin{array}{llll}
-0.36 & -0.6514 & -1.78 & -5.33
\end{array}\right] \\
& K_{31}=\left[\begin{array}{llll}
-0.325 & -0.74 & -2.25 & -2.145
\end{array}\right] \\
& K_{32}=\left[\begin{array}{llll}
-0.245 & -0.478 & -2.98 & -3.916
\end{array}\right] \\
& K_{41}=\left[\begin{array}{llll}
-0.825 & -1.88 & -5.72 & -5.45
\end{array}\right] \\
& K_{24}=K_{34}=K_{42}=K_{43}=0
\end{aligned}
$$

And the overall gain matrix can be calculated as in (31) to be 
TABLE 3: Calculated weighting matrices required to shift area poles into desired new locations.

\begin{tabular}{|c|c|c|c|c|c|c|c|}
\hline & $\begin{array}{c}\text { Nominal pole } \\
\text { locations }\end{array}$ & $\begin{array}{c}\text { Shifted pole } \\
\text { locations }\end{array}$ & \multicolumn{5}{|c|}{ Weighting matrix $Q$} \\
\hline \multirow{6}{*}{ Area2 } & & & \multirow{5}{*}{$\widetilde{Q}_{2}=$} & 1 & 0 & 0 & 0 \\
\hline & -14.315 & -14.315 & & 0 & 1773.63 & 0 & 0 \\
\hline & $-0.447 \pm j 2.84$ & $-4 \pm j 2$ & & & 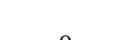 & & \\
\hline & -1.75 & -20 & & 0 & 0 & 11.146 & 0 \\
\hline & & & & 0 & 0 & 0 & 11.146 \\
\hline & & & \multirow{4}{*}{$\widetilde{Q}_{3}=$} & 1 & 0 & 0 & 0 \\
\hline \multirow{4}{*}{ Area3 } & -14.844 & -14.844 & & 0 & 133906.1 & 0 & 0 \\
\hline & $\begin{array}{c}-0.4164 \pm j 3.49 \\
-1.516\end{array}$ & $\begin{array}{c}-4.1 \pm j 2.95 \\
-20\end{array}$ & & 0 & 0 & 76.385 & 0 \\
\hline & & & & 0 & 0 & 0 & 76.385] \\
\hline & & & \multirow{5}{*}{$\widetilde{Q}_{4}=$} & 1 & 0 & 0 & 0 \\
\hline \multirow{4}{*}{ Area 4} & -12.69 & -12.69 & & 0 & $8.337 * 10^{6}$ & 0 & 0 \\
\hline & $-0.4986 \pm j 3.55$ & $-4 \pm j 3$ & & & & & \\
\hline & -0.8084 & -20 & & 0 & 0 & 51.966 & 0 \\
\hline & & & & 0 & 0 & 0 & 51.966 \\
\hline
\end{tabular}

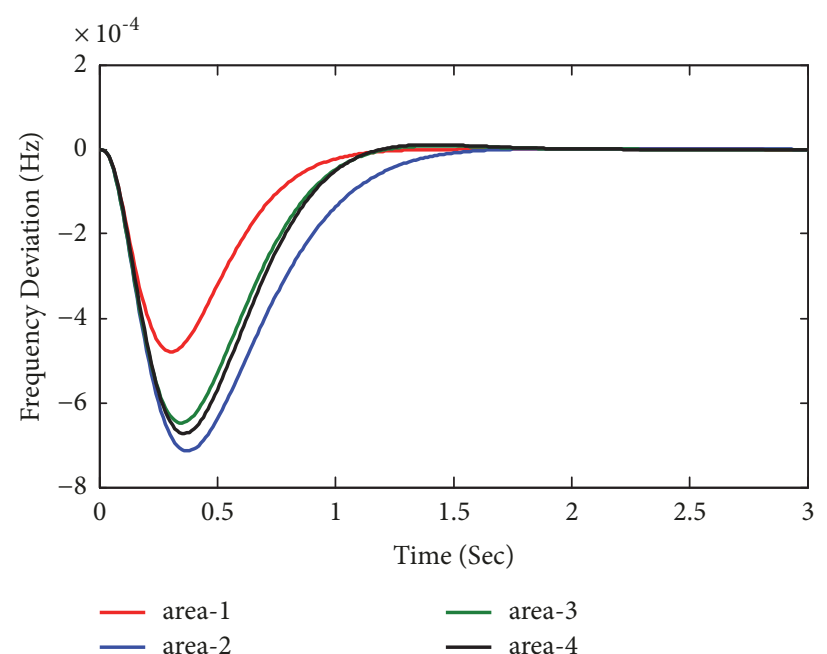

(a)

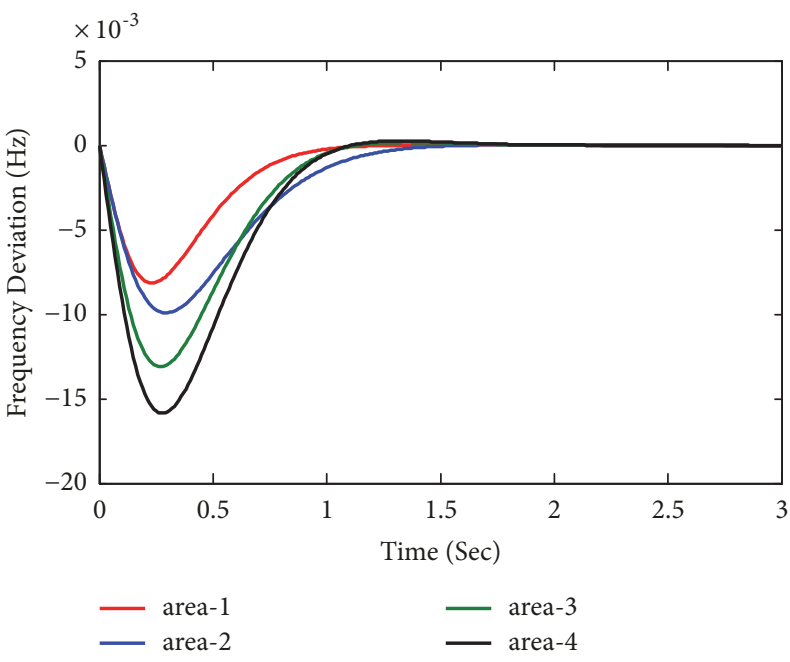

(b)

FIGURE 5: Step response of the controlled system with LQR applied over subsystem level for (a) 0.01 p.u. input and (b) 0.01 disturbance input.

K

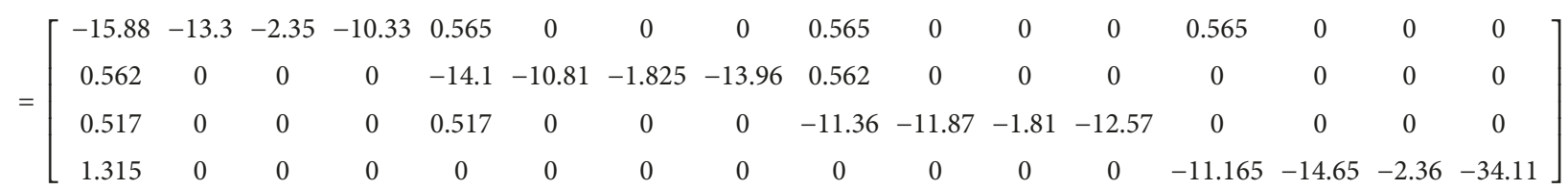

System step responses to 0.01 p.u input and 0.01 p.u disturbance are obtained and illustrated in Figure 5

To check the robustness of this design, it is required to find the controlled system step responses for $\pm 40 \%$ uncertainties with and without disturbances. Figures 6 and 7 illustrate these responses.

All the controlled system specifications are calculated from Figures 5, 6, and 7 and summarized in Table 4. 


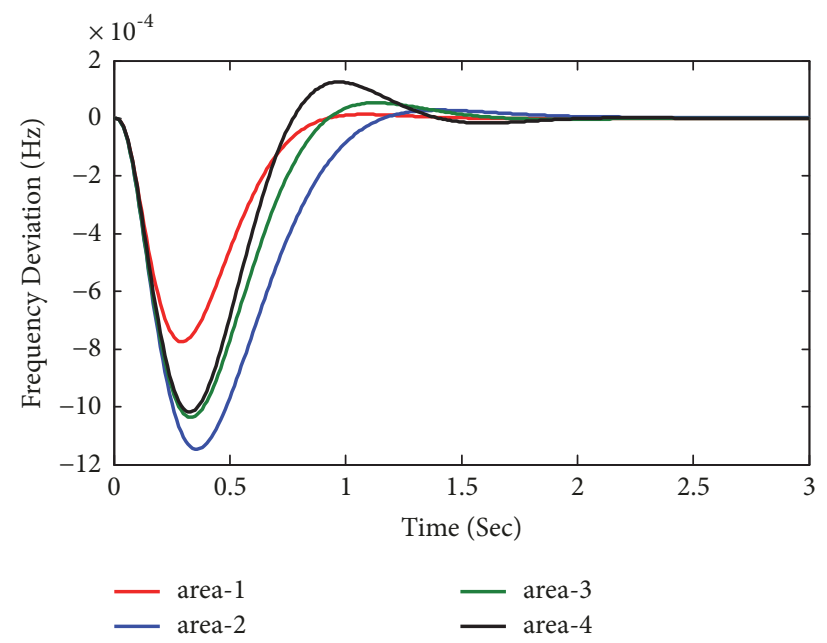

(a)

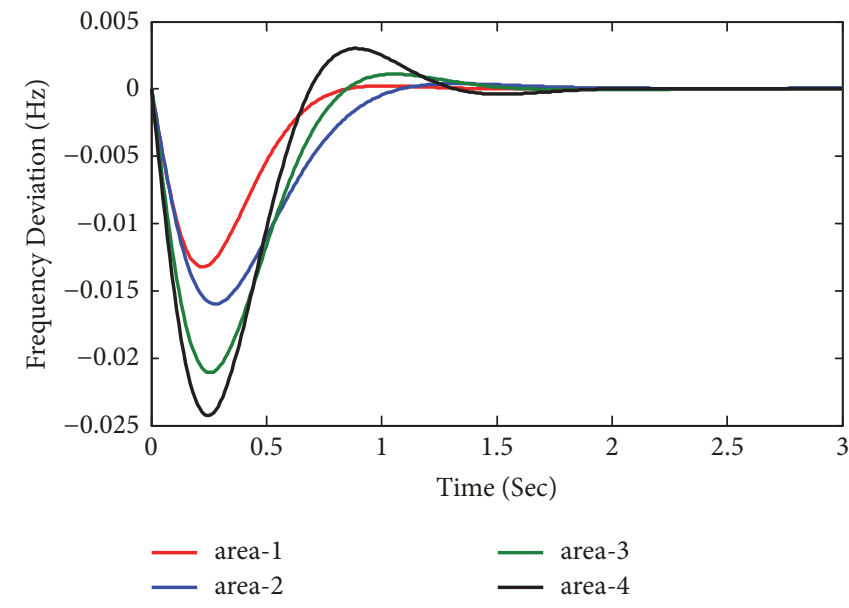

(b)

FIGURE 6: Step response of the four areas with $-40 \%$ uncertainties of $M_{i}$, and $T_{i j}$. (a) for 0.01 p.u input and (b) for 0.01 p.u disturbance.

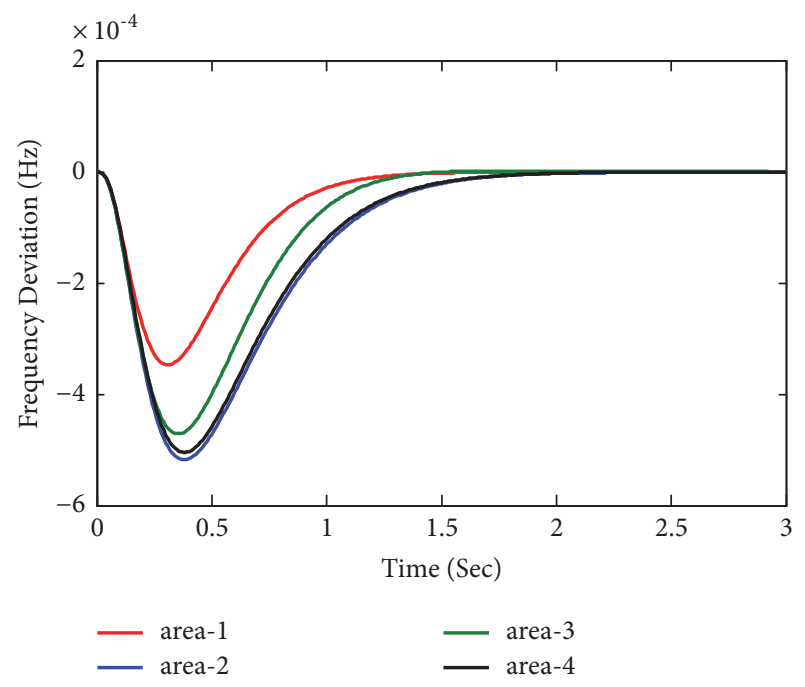

(a)

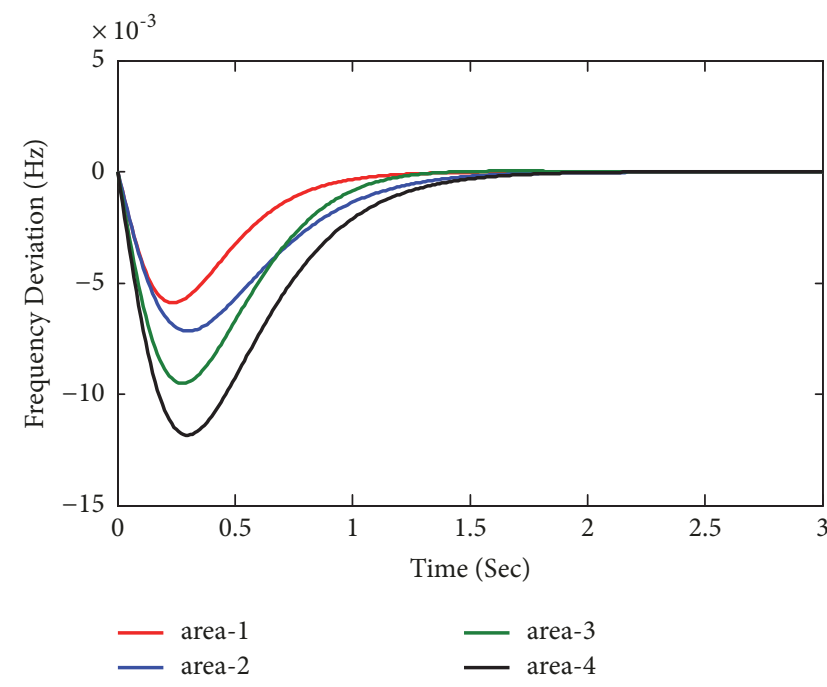

(b)

FIgURE 7: Step response of the four areas with $+40 \%$ uncertainties of $M_{i}$, and $T_{i j}$ (a) for 0.01 p.u input and (b) for 0.01 p.u disturbance.

TABLE 4: Controlled system specifications for scenario II.

\begin{tabular}{|c|c|c|c|c|c|c|}
\hline & \multicolumn{2}{|c|}{ Controlled System } & \multicolumn{2}{|c|}{$\begin{array}{c}\text { Controlled System With }-40 \% \\
\text { Uncertainties }\end{array}$} & \multicolumn{2}{|c|}{$\begin{array}{c}\text { Controlled System With }+40 \% \\
\text { Uncertainties }\end{array}$} \\
\hline & 0.01 p.u Input & $\begin{array}{c}0.01 \text { p.u } \\
\text { Disturbance }\end{array}$ & 0.01 p.u Input & $\begin{array}{c}0.01 \text { p.u } \\
\text { Disturbance }\end{array}$ & 0.01 p.u Input & $\begin{array}{c}0.01 \text { p.u } \\
\text { Disturbance }\end{array}$ \\
\hline$M_{p p}$ & 0 & 0 & 0.000126 & 0.00301 & 0 & 0 \\
\hline$t_{p p}$ & - & - & $0.97 \mathrm{sec}$ & $0.88 \mathrm{sec}$ & - & -- \\
\hline$M_{n p}$ & 0.000714 & 0.01586 & 0.001147 & 0.0243 & 0.00052 & 0.01184 \\
\hline$t_{n p}$ & $0.37 \mathrm{sec}$ & $0.28 \mathrm{sec}$ & $0.36 \mathrm{sec}$ & $0.24 \mathrm{sec}$ & $0.39 \mathrm{sec}$ & $0.3 \mathrm{sec}$ \\
\hline$t_{s}$ & $1.61 \mathrm{sec}$ & $1.52 \mathrm{sec}$ & $1.89 \mathrm{sec}$ & $1.7 \mathrm{sec}$ & $1.82 \mathrm{sec}$ & $1.71 \mathrm{sec}$ \\
\hline
\end{tabular}


TABLE 5: Nominal and controlled overall system eigenvalues.

\begin{tabular}{lccc}
\hline & & Design Scenario & Two Level Control \\
Nominal System & I & II & -34.36 \\
-14.844 & -34.55 & -21.966 & $-21.42 \pm j 1.508$ \\
-14.314 & -17.7 & -20.53 & -21.5 \\
-13.26 & -15.1 & $-18.2 \pm j 3.078$ & -20.108 \\
-12.69 & -13.56 & $-18 \pm 1.62$ & -16.58 \\
$-0.238 \pm j 4.456$ & $-0.606 \pm j 4.335$ & -11.46 & $-15.586 \pm j 6.03$ \\
$-0.336 \pm j 3.696$ & $-0.955 \pm j 3.258$ & $-6.47 \pm j 6.13$ & -12.7 \\
$-0.57 \pm j 3.2897$ & $-1.215 \pm j 2.9$ & -3.45 & $-5.61 \pm j 6.15$ \\
$-1.133 \pm j 2.236$ & $-1.782 \pm j 0.925$ & $-3.346 \pm j 4.38$ & $-4.63 \pm j 4.69$ \\
-2.082 & $-1.0435 \pm j 0.321$ & $-3.45 \pm j 5.577$ & -4 \\
-1.877 & -1.86 & $-1 * 10^{-15}$ & -7.03 \\
-0.9124 & $-17 * 10^{-15}$ & & $-4.39 * 10^{-7}$ \\
$-1 * 10^{-15}$ & & & \\
\hline
\end{tabular}

\section{Result Discussions}

For Scenario I. Figure 3 shows that all areas are stable with more oscillations and have both over and under shoot values. The overall system eignvalues can also be calculated and illustrated in Table 5. Figure 4 shows that area-2 becomes unstable. Time responses for $40 \%$ increasing uncertainties ensure that area- 2 becomes unstable; in addition, the responses for other areas become more oscillatory. Therefore, this design is not a strong design.

For Scenario II. System step responses for nominal values (Figure 5 ) and for $\pm 40 \%$ uncertainties with the presence of load disturbance (Figures 6 and 7) and the numerical data in Table 4 ensure that the system is strong and stable with satisfied specifications. Overall system eigenvalues are listed in Table 5.

The design using LQR with a prespecified eigenvalues is a strong design with fast response and zero frequency deviation.

$$
\begin{aligned}
& K_{\text {levelII }} \\
& =\left[\begin{array}{cccccccc}
0.043 & -0.16 & 26.55 & -3.54 & -0.375 & -0.866 & -7.2 & -11.043 \\
-0.272 & -0.804 & -3.12 & -2.46 & 0.13 & -0.036 & 26.83 & 4.055 \\
0.16 & 0.504 & 1.4 & 5.43 & 0.115 & 0.305 & 1.44 & 8.52 \\
0.055 & 0.146 & 0.111 & 2.83 & 0.076 & 0.21 & 1.93 & 4.81
\end{array}\right.
\end{aligned}
$$

For the computed overall system eignvalues in Table 5, it is noted that all of them are moved into more stable places. A much more shifting to overall system eigenvalues can be obtained if the slower eigenvalues of each subsystem are moved farther inside the stability region but with much more control effort.

A better performance is obtained by the proposed method compared to that obtained by [3]. Smaller settling time, smaller overshoot, and undershoot values with the same zero frequency deviation for time responses to 0.01
Second Controller Level. Table 5 ensures that because of shifting the slowest eigenvalues of the individual areas, most of the overall system eigenvalues are shifted more inside the stability region except one slow pole still as it is with local controllers. When global controllers are designed, there is no more important change over the resulting overall system eigenvalues and to enhance these locations apply LQR technique again but over the aggregated system level.

Use the overall weighting matrix $Q$ of the form

$$
Q=\left[\begin{array}{ccc}
Q_{(1,1)} & \cdots & 0 \\
\vdots & \ddots & \vdots \\
0 & \cdots & Q_{(16,16)}
\end{array}\right]
$$

After many trials, weighting matrix elements can be selected at $Q_{(3,3)}=1000, Q_{(4,4)}=1551, Q_{(7,7)}=1500, Q_{(8,8)}=1000$, and the rest of the diagonal values are equal to 1 , with $R=1$.

The additional required overall gain is p.u load disturbance in the presence of $\pm 40 \%$ change in the same uncertain parameters. Also, this design gets a better performance for frequency deviation to load disturbance than that in $[4,21]$.

\section{Conclusion}

In this paper an optimal controller based LQR is proposed to design a decentralized load frequency controller to an interconnected 4-area power system and compared to that 
designed by other researchers. This method succeeds to realize the design objectives. It moves the undesired subsystem eigenvalues into more stable prespecified ones which in turn shifts the overall system pole locations more inside the stability region and enhances system internal stability.

The simulation results demonstrate the effectiveness of the presented method to provide a robust frequency regulator when the system is affected by $\pm 40 \%$ uncertainties. A zerosteady state frequency deviation is obtained for each area with accepted settling time, overshoot, and undershoot values; also the worst-case performance is accepted. The internal stability is enhanced when LQR is applied again over the overall controlled system level.

\section{Conflicts of Interest}

The authors declare no conflicts of interest.

\section{References}

[1] Y. Zheng, J. Zhou, Y. Xu, Y. Zhang, and Z. Qian, "A distributed model predictive control based load frequency control scheme for multi-area interconnected power system using discrete-time Laguerre functions," ISA Transactions, vol. 68, pp. 127-140, 2017.

[2] S. K. Pandey, S. R. Mohanty, and N. Kishor, "A literature survey on load-frequency control for conventional and distribution generation power systems," Renewable \& Sustainable Energy Reviews, vol. 25, pp. 318-334, 2013.

[3] H. Shayeghi, "A robust decentralized power system load frequency control," Journal of Electrical Engineering, vol. 59, no. 6, pp. 281-293, 2008.

[4] S. Selvakumarana, S. Parthasarathy R, and V. Rajasekaran, "Optimal decentralized load frequency control in a parallel ACDC Interconnected power system through HVDC link using PSO algorithm," Energy Procedia, vol. 14, pp. 1849-1854, 2012.

[5] D. Thi-Mai-Phuong, Y. Wang, and N. Ngoc-Khoat, "Novel hybrid load-frequency controller applying artificial intelligence techniques integrated with superconducting magnetic energy storage devices for an interconnected electric power grid," Arabian Journal for Science and Engineering, vol. 41, no. 9, pp. 3309-3320, 2016.

[6] S. A. Taher, R. Hematti, A. Abdolalipour, and S. H. Tabei, “Optimal decentralized load frequency control using HPSO algorithms in deregulated power systems," American Journal of Applied Sciences, vol. 5, no. 9, pp. 1167-1174, 2008.

[7] H. Cimen, "Decentralised $\mathrm{H}_{\text {inf }}$ load frequency controller design based on SSVs," Turkish Journal of Electrical Engineering and Computer Science, vol. 8, no. 1, 2000.

[8] T. Yang, Z. Ding, and H. Yu, "Decentralised power system load frequency control beyond the limit of diagonal dominance," International Journal of Electrical Power \& Energy Systems, vol. 24, no. 3, pp. 173-184, 2002.

[9] H. Bevrani, Y. Mitani, and K. Tsuji, "Sequential decentralized design of robust load frequency controllers in multiarea power systems," IFAC Proceedings Volumes, vol. 36, no. 20, pp. 109-114, 2003.

[10] H. Bevrani, Y. Mitani, and K. Tsuji, "Sequential design of decentralized load frequency controllers using $\mu$ synthesis and analysis," Energy Conversion and Management, vol. 45, no. 6, pp. 865881, 2004.
[11] H. Bevrani, Robust Power System Frequency Control, Springer Science and Business Media, LLC, 2009.

[12] M. Elsisi, M. Soliman, M. A. S. Aboelela, and W. Mansour, "Optimal design of model predictive control with superconducting magnetic energy storage for load frequency control of nonlinear hydrothermal power system using bat inspired algorithm," Journal of Energy Storage, vol. 12, pp. 311-318, 2017.

[13] O. A. Solheim, "Design of optimal control systems with prescribed eigenvalues," International Journal of Control, vol. 15, no. 1, pp. 143-160, 1972.

[14] V. Kucera and J. Cigler, "Optimal control systems with prescribed eigenvalues," in Proceedings of the 4th International Symposium on Communications, Control and Signal Processing (ISCCSP 2010), pp. 1-6, Limassol, Cyprus, March 2010.

[15] S. A. Ghoreishi, M. A. Nekoui, and S. O. Basiri, "Optimal design of LQR weighting matrices based on intelligent optimization methods," International Journal of Intelligent Information Processing, vol. 2, no. 1, pp. 63-74, 2011.

[16] R. M. Stefanescu, C. L. Prioroc, and A. M. Stoica, "Weighting matrices determination using pole placement for tracking maneuvers," UPB Scientific Bulletin, Series D: Mechanical Engineering, vol. 75, no. 2, pp. 31-40, 2013.

[17] A. Mustafa, K. Munawar, F. M. Malik, M. B. Malik, M. Salman, and S. Amin, "Reduced order observer design with DMPC and LQR for system with backlash nonlinearity," Arabian Journal for Science \& Engineering, vol. 39, no. 8, pp. 6521-6530, 2014.

[18] D. D. Šiljak and M. B. Vukčević, "Decentralization, stabilization, and estimation of large-scale linear systems," IEEE Transactions on Automatic Control, vol. 21, no. 3, pp. 363-366, 1976.

[19] B. Labibi, B. Lohmann, A. Sedigh, and P. Maralani, "Sufficient condition for stability of decentralised control," IEEE Electronics Letters, vol. 36, no. 6, p. 588, 2000.

[20] V. Kučera, "Decoupling optimal controllers," in Proceedings of the 18th International Conference on Process Control, pp. 400407, Tatranská Lomnica, Slovakia, 2011.

[21] N. Kumar, B. Tyagi, and V. Kumar, "Deregulated Multiarea AGC Scheme Using BBBC-FOPID Controller," Arabian Journal for Science and Engineering, vol. 42, no. 7, pp. 2641-2649, 2017. 


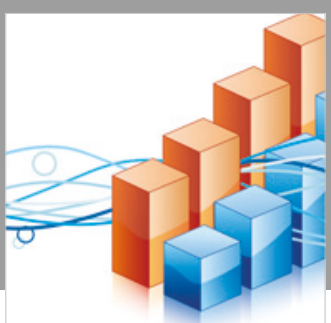

Advances in

Operations Research

\section{-n-m}
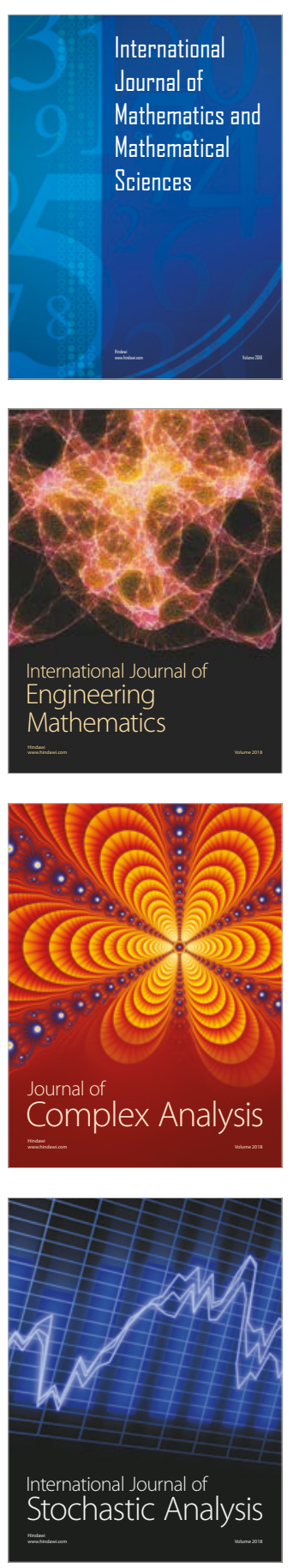
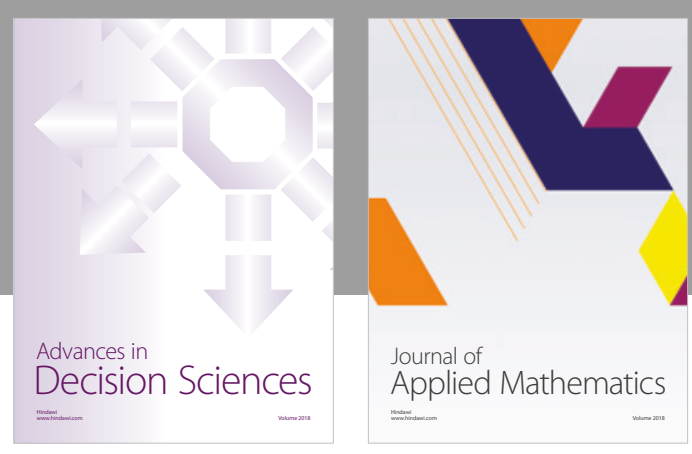

Journal of

Applied Mathematics
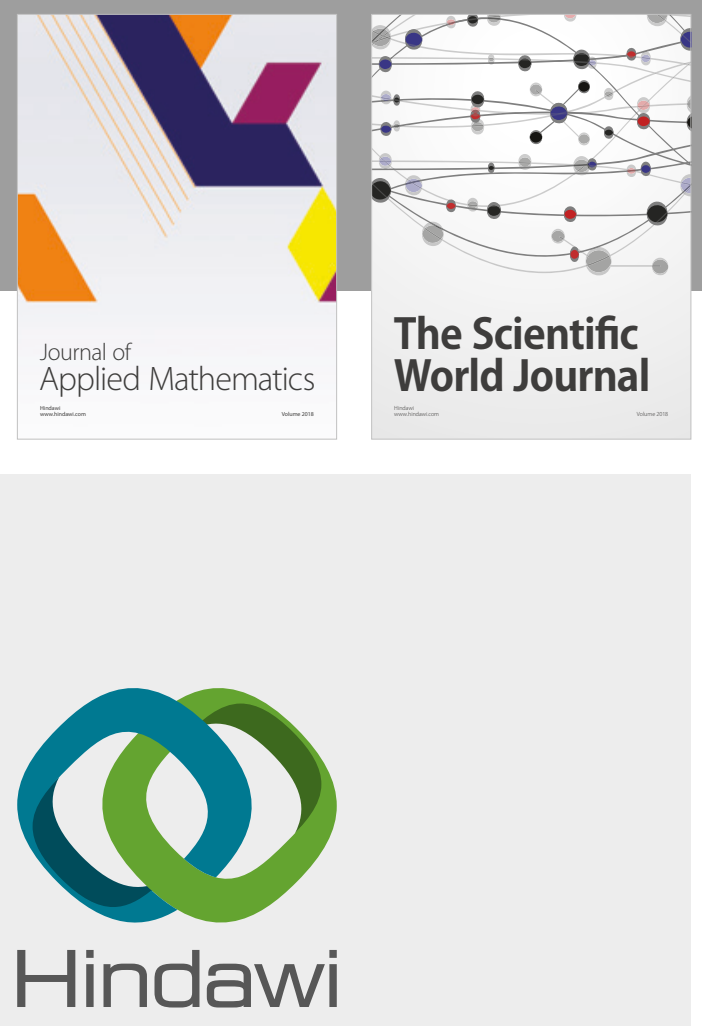

Submit your manuscripts at

www.hindawi.com

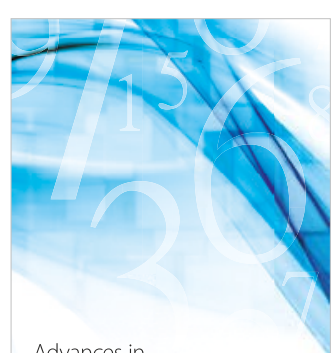

Advances in
Numerical Analysis
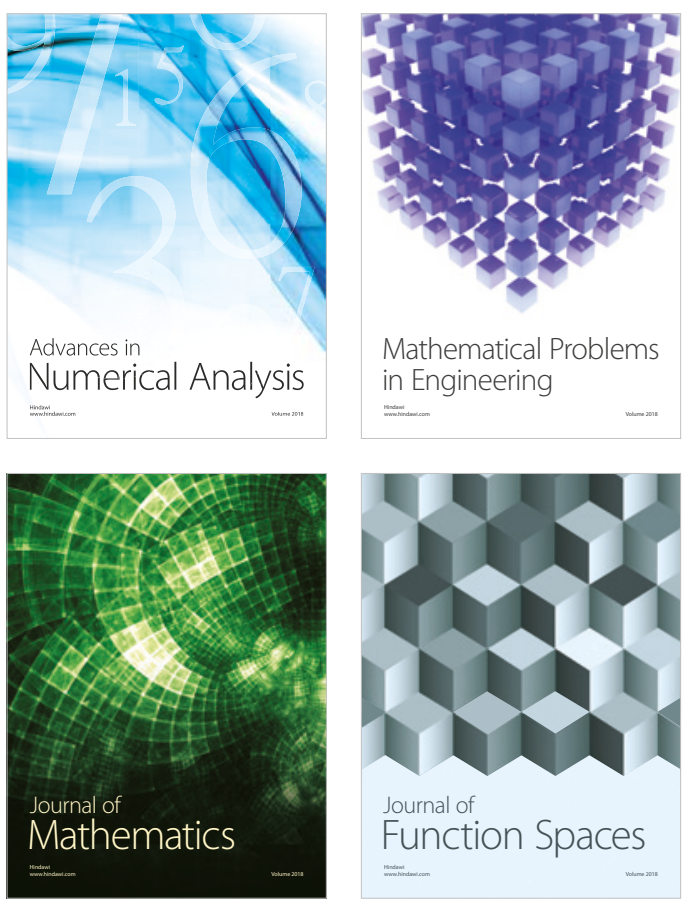

Mathematical Problems in Engineering

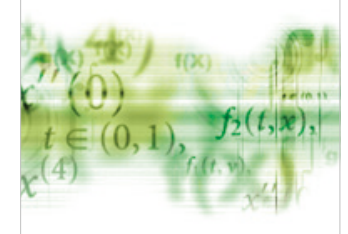

International Journal of

Differential Equations

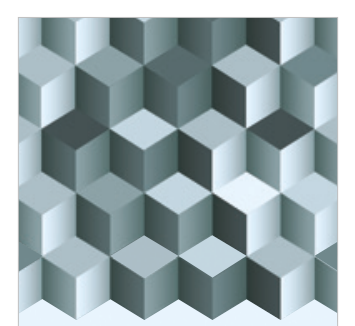

Journal of

Function Spaces

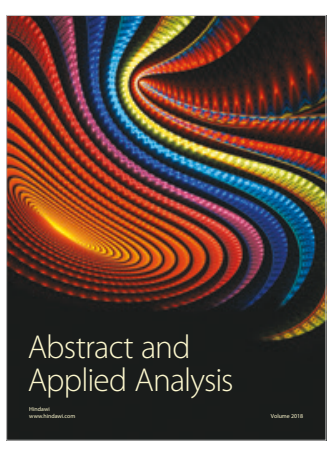

The Scientific

World Journal

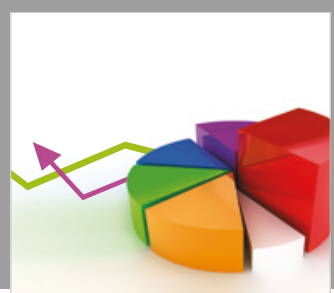

Journal of

Probability and Statistics
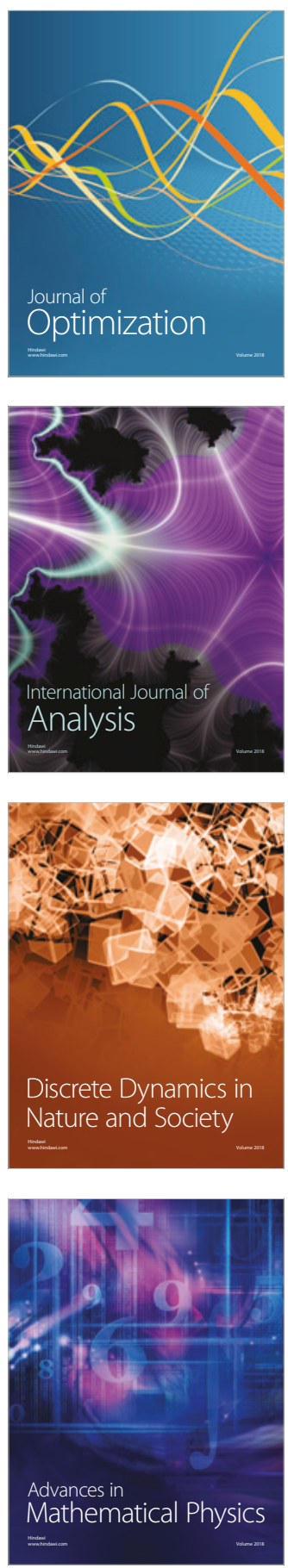